\title{
Acute demyelinating encephalomyelitis and transverse myelitis in a child with COVID-19
}

\author{
Hatice Gamze Poyrazoğlu1 ${ }^{1 \oplus}$, Serkan $\mathrm{K}_{1 r 1 k}{ }^{1 \oplus}$, Mehmet Yusuf Sar1 ${ }^{2 \oplus}$, İhsan Esen ${ }^{3 \odot}$, \\ Zülal Aşçı Toraman ${ }^{4 \oplus}$, Yeşim Eroğlu ${ }^{5 \oplus}$ \\ Divisions of ${ }^{1}$ Pediatric Neurology and ${ }^{2}$ Pediatric Intensive Care Unit, ${ }^{3}$ Department of Pediatrics, Departments of ${ }^{4}$ Microbiology and \\ ${ }^{5}$ Radiology, Firat University Faculty of Medicine, Elazı̆̆, Turkey.
}

\begin{abstract}
Background. Corona virus disease 2019 (COVID-19) includes a wide range of diseases with varying pathophysiology in children and adults. Although the disease mainly affects the respiratory tract, neurological involvement is also reported in the literature. The most common neurological complaints due to COVID-19 are headache, dizziness and anosmia. Acute necrotizing myelitis, acute demyelinating encephalomyelitis (ADEM), acute axonal neuropathy, acute transverse myelitis, and Guillian-Barre syndrome have been reported as neurological dysfunctions associated with COVID-19.
\end{abstract}

Case. A ten-year-old male patient presented with complaints of fever, headache and generalized muscle pain. The patient developed inability to walk and significant muscle weakness during the disease course, and he was diagnosed with ADEM and transverse myelitis on magnetic resonance imaging (MRI). As the etiological agent, COVID-19 was detected in both the respiratory panel sample and the cerebrospinal fluid (CSF) sample by the polymerase chain reaction (PCR) technique. Pulse steroid, IVIG, and plasmapheresis treatment were administered. He started to stand with support during follow-up.

Conclusion. We presented a case of COVID-19 related ADEM and transverse myelitis who responded to pulse steroid, IVIG, and plasmapheresis

Key words: acute demyelinating encephalomyelitis, acute transverse myelitis, COVID-19.

Infection associated with COVID-19, which emerged in Wuhan, China in December 2019, spread rapidly all over the world. ${ }^{1}$ SARS-Cov 2 infection can classically lead to fever, cough, diarrhea, generalized muscle aches, severe respiratory disease and severe respiratory failure that may progress to acute respiratory distress syndrome (ARDS). ${ }^{2}$ The diagnosis of COVID-19 disease is made by viral nucleic acid detection by molecular methods and abnormal livermetabolic tests supported by hematological panels. ${ }^{3,4}$ Although the disease mostly affects the respiratory tract, neurological involvement has also been reported in the literature. The invasion

Hatice Gamze Poyrazoğlu
hgpoyrazoglu@yahoo.com

Received 13th November 2020,

revised 28th December 2020, accepted 8th February 2021. of the central nervous system with COVID-19 occurs completely through immune escape and shortly after infection. The virus enters the central nervous system and causes a series of disease-related consequences. ${ }^{5}$ Neurological involvement characteristics of this infection include conditions such as headache, dizziness, epileptic seizures, taste and smell disorders, cerebrovascular events, acute encephalitis, Guillain-Barre syndrome, and acute transverse myelitis. ${ }^{6,7}$ Researchers have confirmed that the SARS-CoV-2 virus enters cells through the angiotensin-converting enzyme (ACE) 2 receptors on the surface of human cells and causes the disease. ${ }^{8}$ ACE2 receptors found in type 2 alveolar epithelial cells of human lung have become the main target of SARS-CoV-2 virus in the pathogenesis of COVID-19. ${ }^{9}$ In addition to highly marked lung, liver and kidney 
injuries possibly associated with the expression of ACE 2 in proximal renal tubules and hepatic bile duct cells, many critical patients with multiorgan dysfunction have been reported during the clinical diagnosis and treatment process. ${ }^{10,11}$ ACE receptors are also found on the surface of spinal cord cells. However, it is still not entirely clear whether neurons of the spinal cord are adversely affected by COVID-19.12,13 In this report, we present a 10-year-old male patient with acute demyelinating encephalomyelitis (ADEM) and transverse myelitis, started with fever and headache and continued with weakness and inability to walk later in the course, which was found to be associated with COVID-19.

\section{Case Report}

A 10-year-old male patient, who had been healthy until a month prior, had presented to a physician with weakness, vomiting, severe headache, and fever; he had been treated for sinusitis. A brain magnetic resonance imaging (MRI) study had been performed and found normal. One week after the treatment, the patient had presented to the physician again due to the persistence of headache, severe coliclike abdominal pain attacks, and widespread body pain. He was hospitalized and received ceftriaxone. On the 8th day of treatment, he started to be agitated and have tremors in his left arm and leg. Later in the course, he was unable to walk and use his extremities. The patient whose general condition deteriorated was referred to our pediatric emergency department. At emergency department admission, he had a body temperature of $36 .{ }^{\circ} \mathrm{C}$, a blood pressure reading of $100 / 70$ $\mathrm{mmHg}$, and a pulse rate of 95/min.; his general condition was moderate-to-poor; he was somnolent, got occasionally agitated and was crying. His pupils were isochoric with positive diagnostic likelihood ratio (DLR) \likelihood ratio (LR), and he had marked neck stiffness. He had positive meningeal irritation signs. He had marked clonus in both lower extremities. His abdominal skin reflex was absent in all abdominal quadrants. His muscle strength was $0 / 5$ in the left upper extremity, $2 / 5$ in the right upper extremity, and $3 / 5$ in both lower extremities. His laboratory tests revealed a WBC of $21,800 / \mathrm{mm}^{3}$ with a PMNL predominance of $\% 92.5$ and a platelet count of $358,000 / \mathrm{mm}^{3}$; blood glucose was $124 \mathrm{mg} / \mathrm{dl}$, ALT $18 \mathrm{U} / \mathrm{L}$, urea $54 \mathrm{mg} / \mathrm{dl}$, creatinine $1.66 \mathrm{mg} / \mathrm{dl}$; the coagulation tests were normal; he had a sedimentation rate of $38 \mathrm{~mm}$ /hour and a negative $\mathrm{C}$-reactive protein (CRP); the urinalysis showed a urinary $\mathrm{pH}$ of 6.5 , urine density of 1007 , negative urinary protein and glucose, trace erythrocytes, and a leucocyte count of $19.5 / \mathrm{hpf}$. Urine culture was sterile. The patient was admitted to the intensive care unit and meropenem, acyclovir, and clarithromycin were started. The patient was hydrated and his fluid intake and urine output were monitored. His renal function improved during follow-up. Brain and cervical MRI was performed and revealed marked signal increase in basal ganglia and cervical myelitis (Fig.1 a, $b, e)$. Lumbar puncture (LP) was delayed due to his poor general condition. Intravenous methylprednisolone was administered at a dose of $30 \mathrm{mg} / \mathrm{kg} /$ day for 5 days. The patient's general condition slightly improved on the third day of methylprednisolone treatment, and thus LP was performed. Cerebrospinal fluid (CSF) pressure was within normal limits, CSF was clear, and no cells were seen. CSF protein level was $38.3 \mathrm{mg} / \mathrm{dl}$, and CSF glucose level was $89 \mathrm{mg} / \mathrm{dl}$. There was no growth in CSF culture or blood cultures. Polymerase chain reaction (PCR) tests for Neisseria meningitidis, Hemophilus influenzae, Streptococcus pneumoniae, varicella zoster, herpes simplex virus type 1 and type 2 in the CSF were all negative. No findings other than 2019 ncov PCR positivity was detected in the respiratory panel studied from the nasal swab. Therefore, the 2019 ncov PCR test was reexamined in the CSF sample. As the 2019 ncov PCR test was positive in the CSF, it was thought that the patient possibly had ADEM + transverse myelitis secondary to COVID-19. Methylprednisolone was administered at a dose of $30 \mathrm{mg} / \mathrm{kg} /$ day for 5 days, followed by a dose of $30 \mathrm{mg}$ twice a day, after that plasmapheresis was 
performed for five days, and then intravenous immunoglobulin (IVIG) was administered at a dose of $400 \mathrm{mg} / \mathrm{kg}$. It was planned that steroid treatment was administered at a dose of $2 \times 30 \mathrm{mg}$ for 4 weeks and tapered in 4-6 weeks. The chest radiography of the patient was normal. The patient was not administered direct treatment for COVID-19. Oligoclonal band, serum antimog and aquaporin 4 antibodies in CSF were negative. On the 5th day of plasmapheresis and IVIG treatment, the muscle strength of the patient's left upper extremity was 3-4/5; although his neck stiffness was significantly reduced, it persisted. The patient's clinical status significantly improved. At the end of the
IVIG + plasmapheresis treatment, the brain axial T2 and DIR sequences of the control brain and spinal MRI detected regression in the findings (Fig.1 c, d, f).

Informed consent was received from the family.

\section{Discussion}

Pediatric acute transverse myelitis is an immune-mediated central nervous system disease. ${ }^{14-17}$ Transverse myelitis is a heterogeneous, non-compressive myelopathy characterized by acute- or subacute-onset spinal cord dysfunction due to inflammation. ${ }^{15}$
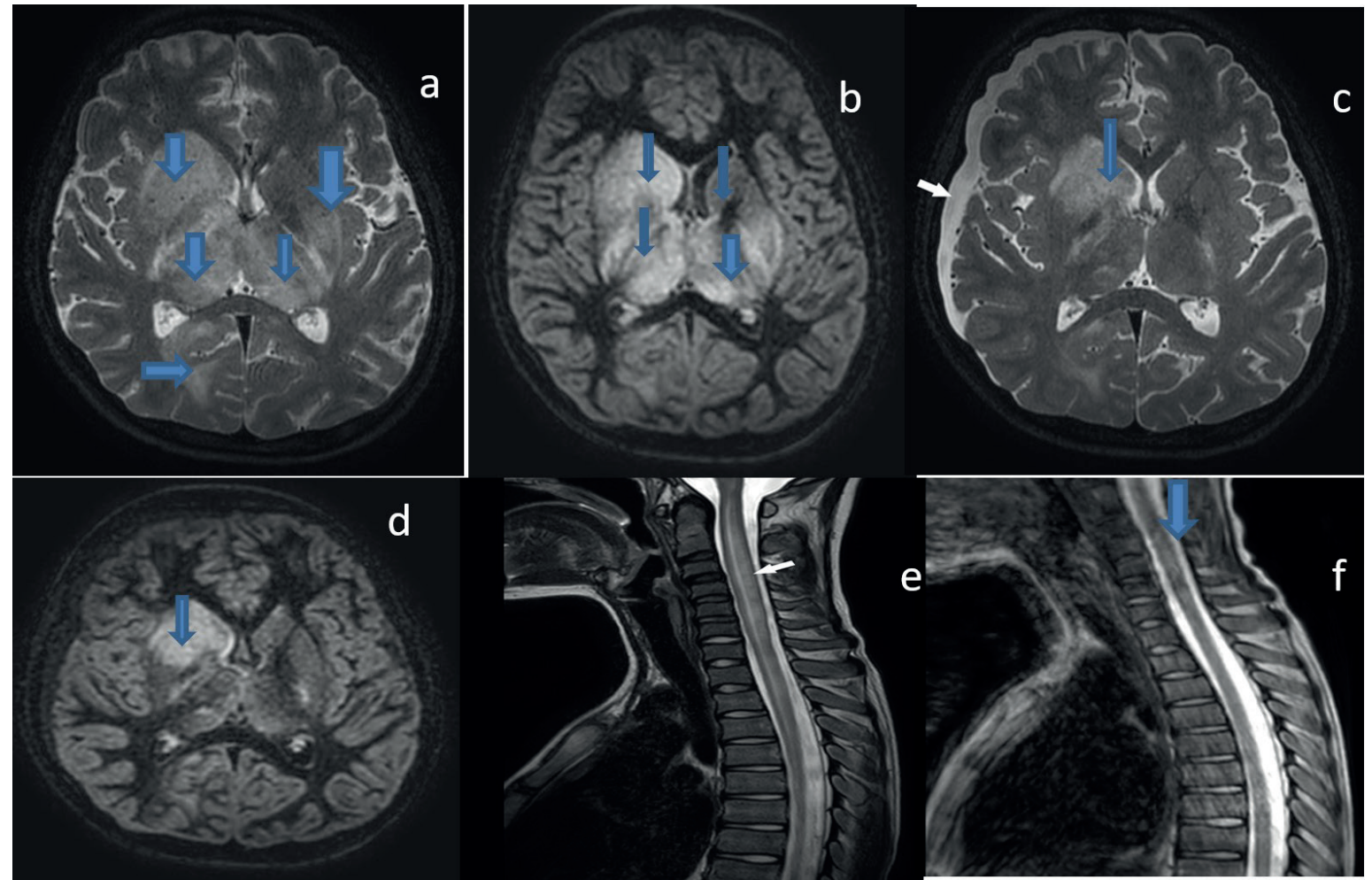

Fig. 1. a-b) T2 axial and axial double inversion recovery (DIR) sequences of brain magnetic resonance imaging (MRI) study revealed no contrast enhancement or diffusion limitation, but marked signal increase, in the right caudate nucleus, bilateral lentiform nuclei, bilateral thalami, partially internal and external capsules, both occipital lobes (predominantly right side) and left hippocampus, and bilateral amygdala (blue arrow). c-d) Axial T2 and DIR sequences of the control brain MRI detected regression in the findings, being more pronounced in the bilateral thalamus (blue arrow). In addition, a newly developed subdural effusion (white arrow) was observed on the right side. e) On cervical MRI, there was a central signal increase (myelitis?) of a slightly expansive character in the cervicothoracal spinal cord (white arrow), which was more prominent in the cervical region; there was only millimetric punctate enhancement at the $\mathrm{C} 4$ level, but no enhancement was observed in other parts. f) Sagittal T2 sequences spinal cord expansion signal increase regressed in post-treatment control spinal MRI (blue arrow). 
The clinical signs include neurological deficits characterized by motor, autonomic, and sensory involvement in varying degrees. Acute demyelinating diseases such as para-infectious, post-infectious, toxic-drug-related causes, paraneoplastic, autoimmune disorders, and multiple sclerosis and neuromyelitis optica spectrum disorders are held responsible for the etiology of ATM. ${ }^{14}$ Acute demyelinating encephalomyelitis (ADEM) is a common postviral demyelinating disease that is mostly seen in children rather than adults. Although clinically heterogeneous, it causes multifocal deficits and encephalopathy. Brain MRI FLAIR images typically show hyperintensity in deep white matter and at the gray-white matter interface. Contrast enhancement usually does not occur, but rarely, punctate contrast enhancement can be seen. Diffusion restriction can also be seen in the early period. ${ }^{18}$

A few cases with neurological findings associated with COVID-19 have been reported in the literature. Acute transverse myelitis was reported for the first time in a 66-year-old Chinese patient with SARS-CoV-2 infection, who presented with acute weakness, urinary and bowel incontinence. ${ }^{19}$ A 59-year-old female patient with high fever was admitted to the emergency room with acute onset of ascending flaccid paraplegia in lower extremities, urinary retention, and constipation; she was diagnosed with acute transverse myelitis associated with COVID-19. ${ }^{14}$ In another case, a 69-year-old female patient presented to a physician with pain in the cervical region, imbalance, motor weakness, and numbness in the left hand 8 days after the onset of fever and dry cough. The patient's spinal MRI showed an appearance compatible with acute transverse myelitis. Although COVID-19 was detected in the sample taken from the respiratory tract of the patient, it was reported that the COVID-19 test was negative in the CSF sample..$^{20}$ The pediatric case in the literature is a 3-year-old girl from Navajo. She presented to hospital with progressive weakness in extremities and reduced sensation. In her examination, she had flask quadriparesia, areflexia, and respiratory failure. Her MRI showed an appearance compatible with transverse myelitis in the cervical region. The patient's nasopharyngeal SARS Cov 2 PCR test was positive. ${ }^{7}$

A 51-year-old female patient was admitted to the hospital with vomiting, dizziness, fever, and difficulty breathing. The SARS cov 2 nasal swab PCR test returned positive. Her deep tendon reflexes were reduced. A contrastenhanced brain MRI showed signs compatible with ADEM. ${ }^{18}$

In conclusion, neurological symptoms due to COVID-19 can be seen in childhood, albeit rarely. We aimed to emphasize that neurological symptoms should be regarded as a post-infectious complication that occurs after COVID-19 or in the absence of specific COVID-19 symptoms and that they can progress rapidly, for which aggressive first-line treatments should be considered. In addition, the co-occurrence of COVID-induced ADEM and acute transverse myelitis was emphasized because it has not been reported in children in the literature.

\section{Author contribution}

The authors confirm contribution to the paper as follows: study conception and design: HGP, SK; data collection: HGP, SK, MYS, IE, ZAT, $\mathrm{YE}$; analysis and interpretation of results: HGP, SK, MYS, IE, ZAT, YE; draft manuscript preparation: HGP, SK,YE. All authors reviewed the results and approved the final version of the manuscript.

\section{Conflict of interest}

The authors declare that there is no conflict of interest. 


\section{REFERENCES}

1. Zhu N, Zhang D, Wang W, et al; China Novel Coronavirus Investigating and Research Team. A novel coronavirus from patients with pneumonia in China, 2019. N Engl J Med 2020; 382: 727-733. https:// doi.org/10.1056/NEJMoa2001017

2. Acharya A, Kevadiya BD, Gendelman HE, Byrareddy $\mathrm{SN}$. SARS-CoV-2 infection leads to neurological dysfunction. J Neuroimmune Pharmacol 2020; 15 : 167-173. https://doi.org/10.1007/s11481-020-09924-9

3. Fu L, Wang B, Yuan T, et al. Clinical characteristics of coronavirus disease 2019 (COVID-19) in China: A systematic review and meta-analysis. J Infect 2020; 80: 656-665. https://doi.org/10.1016/j.jinf.2020.03.041

4. Pan L, Mu M, Yang P, et al. Clinical characteristics of COVID-19 patients with digestive symptoms in Hubei, China: a descriptive, cross-sectional, multicenter study. Am J Gastroenterol 2020; 115: 766773. https://doi.org/10.14309/ajg.0000000000000620

5. McGavern DB, Kang SS. Illuminating viral infections in the nervous system. Nat Rev Immunol 2011; 11: 318-329. https://doi.org/10.1038/nri2971

6. Mao L, Jin $H$, Wang $M$, et al. Neurologic manifestations of hospitalized patients with coronavirus disease 2019 in Wuhan, China. JAMA Neurol 2020; 77: 683-690. https://doi.org/10.1001/ jamaneurol.2020.1127

7. Kaur H, Mason JA, Bajracharya M, et al. Transverse myelitis in a child with COVID-19. Pediatr Neurol 2020; 112: 5-6. https://doi.org/10.1016/j. pediatrneurol.2020.07.017

8. Wan Y, Shang J, Graham R, Baric RS, Li F. Receptor recognition by the novel coronavirus from Wuhan: an analysis based on decade-long structural studies of SARS coronavirus. J Virol 2020; 94: e00127-20. https://doi.org/10.1128/JVI.00127-20

9. Reyfman PA, Walter JM, Joshi N, et al. Single-cell transcriptomic analysis of human lung provides insights into the pathobiology of pulmonary fibrosis. Am J Respir Crit Care Med 2019; 199: 1517-1536. https://doi.org/10.1164/rccm.201712-2410OC

10. MacParland SA, Liu JC, Ma XZ, et al. Single cell RNA sequencing of human liver reveals distinct intrahepatic macrophage populations. Nat Commun 2018; 9: 4383. https://doi.org/10.1038/s41467-01806318-7
11. Wu H, Uchimura K, Donnelly EL, Kirita Y, Morris SA, Humphreys BD. Comparative analysis and refinement of human PSC-derived kidney organoid differentiation with single-cell transcriptomics. Cell Stem Cell 2018; 23: 869-881.e8. https://doi. org/10.1016/j.stem.2018.10.010

12. Nemoto $W$, Yamagata $R$, Nakagawasai $O$, et al. Effect of spinal angiotensin-converting enzyme 2 activation on the formalin-induced nociceptive response in mice. Eur J Pharmacol 2020; 872: 172950. https://doi.org/10.1016/j.ejphar.2020.172950

13. Ogata Y, Nemoto W, Yamagata R, et al. Antihypersensitive effect of angiotensin (1-7) on streptozotocin-induced diabetic neuropathic pain in mice. Eur J Pain 2019; 23: 739-749. https://doi. org/10.1002/ejp.1341

14. Chakraborty U, Chandra A, Ray AK, Biswas P. COVID-19-associated acute transverse myelitis: a rare entity. BMJ Case Rep 2020; 13: e238668. https:// doi.org/10.1136/bcr-2020-238668

15. Deiva K, Absoud M, Hemingway C, et al; United Kingdom Childhood Inflammatory Demyelination (UK-CID) Study and French Kidbiosep Study. Acute idiopathic transverse myelitis in children: early predictors of relapse and disability. Neurology 2015; 84: 341-349. https://doi.org/10.1212/ WNL.0000000000001179

16. Wolf VL, Lupo PJ, Lotze TE. Pediatric acute transverse myelitis overview and differential diagnosis. J Child Neurol 2012; 27: 1426-1436. https:// doi.org/10.1177/0883073812452916

17. De Goede CG, Holmes EM, Pike MG. Acquired transverse myelopathy in children in the United Kingdom-a 2 year prospective study. Eur J Paediat Neurol 2010; 14: 479-487. https://doi.org/10.1016/j. ejpn.2009.12.002

18. Parsons T, Banks S, Bae C, Gelber J, Alahmadi $\mathrm{H}$, Tichauer M. COVID-19-associated acute disseminated encephalomyelitis (ADEM). J Neurol 2020; 267: 2799-2802. https://doi.org/10.1007/s00415020-09951-9

19. Chow CCN, Magnussen J, Ip J, Su Y. Acute transverse myelitis in COVID-19 infection. BMJ Case Rep 2020; 13: e236720. https://doi.org/10.1136/bcr-2020-236720

20. Sotoca J, Rodriguez-Alvarez Y. COVID-19-associated acute necrotizing myelitis. Neurol Neuroimmunol Neuroinflamm 2020; 7: e803. https://doi.org/10.1212/ NXI.0000000000000803 